\title{
Characterization of Aspergillus species based on fatty acid profiles
}

\author{
Marcelo E Fraga ${ }^{+}$, Djalva Maria N Santana' ${ }^{1}$, Mario Jorge Gattiㄹ, Gloria Maria Direito, \\ Lilia R Cavaglieri ${ }^{3}$, Carlos Alberto R Rosa
}

Departamento de Microbiologia e Imunologia Veterinária, 'Departamento de Tecnologia de Alimento, Universidade Federal Rural do Rio de Janeiro, BR 465 Km 07, 23890-000 Seropédica, RJ, Brasil ²Laboratório de Avaliação e Promoção da Saúde Ambiental, Instituto Oswaldo Cruz-Fiocruz, Rio de Janeiro, RJ, Brasil ${ }^{3}$ Departamento de Microbiología e Inmunología, Facultad de Ciencias Exactas, Físico-Químicas y Naturales, Universidad Nacional de Río Cuarto, Río Cuarto, Córdoba, Argentina

Cellular fatty acid (FA) composition was utilized as a taxonomic tool to discriminate between different Aspergillus species. Several of the tested species had the same FA composition and different relative FA concentrations. The most important FAs were palmitic acid (C16:0), estearic acid (C18:0), oleic acid (C18:1) and linoleic acid (C18:2), which represented 95\% of Aspergillus FAs. Multivariate data analysis demonstrated that FA analysis is a useful tool for differentiating species belonging to genus Aspergillus. All the species analyzed showed significantly FA acid profiles $(p<0.001)$. Furthermore, it will be possible to distinguish among Aspergillus spp. in the Flavi Section. FA composition can serve as a useful tool for the identification of filamentous fungi.

Key words: Aspergillus spp. - fungi - taxonomy - fatty acid - multivariate analysis

The whole cell fatty acid (FA) profiles, mainly in bacteria, have been used as a species identification tool (Moss 1981, Veys et al. 1989). The value of FA analysis in yeast taxonomy is of increasing interest; FA composition has been screened and analyzed under several conditions (Menyawi et al. 2000). The genus Aspergillus contains approximately 250 species (Samson \& Varga 2007). Some of these species have been used for many years in the fermentation industry for the production of organic acids and, more recently, for the production of a variety of enzymes and other secondary metabolites. On the other hand, some Aspergillus species produce mycotoxins that cause adverse effects on animal and human health (Samson \& Pitt 2000). Fungal identification has mainly been performed according to morphological criteria. Until recently, these techniques have only been used in fungal taxonomy. Although, fewer different FAs are produced by fungi than by bacteria (Lechevalier \& Lechevalier 1988), these analyses are increasingly used to distinguish different fungi (Welch 1991, Stahl \& Klug 1996, Guarro et al. 1999). In earlier studies of filamentous fungi, no multivariate statistical methods have been used to evaluate the FA. Recently, developed programs for multivariate discriminate analysis have helped to increase the value of fungi FA data in routine taxonomy and identification work (Blomquist et al. 1992, Müller et al. 1994, Stahl \& Klug 1996). However, the FA methodology has not been applied as a taxonomic tool for discriminating amongst Aspergillus spp. The aim of the present work was to investigate the FA composition usage as a taxonomic tool for separating Aspergillus spp.

+Corresponding author: fraga@ufrrj.br

Received 13 May 2008

Accepted 28 July 2008

\section{MATERIALS AND METHODS}

Strains - All fungal strains were obtained from the Coleção Micológica do Departamento de Biologia, Instituto Oswaldo Cruz (CMDB-IOC), Brazil, maintained under lyophilizated conditions. Six Aspergillus spp. were used as a microbiological reference strain: A. caelatus (CMDB 0329, origin: NRRL 25528), A. flavus (CMDB 0335, origin: NRRL 5520), A. fumigatus (CMDB 0153, origin: ATCC 16913), A. nomius (CMDB 0155, origin: NRRL 13137), A. ochraceus (CMDB 0154, origin: ATCC 22947), A. parasiticus (CMDB 0334, origin: ATCC 15517), where the origin corresponds, respectively, to the American Type Culture Collection (ATCC- USA) and the National Center for Agricultural Utilization Research (NRRL-USA). Eighty three A. flavus, nine $A$. parasiticus and four A. tamarii isolated from maize and poultry feed were also used in the study.

The identification of Aspergillus spp. was done with morphological taxonomy, in accordance with the colony diame-ter, conidial color, exudates, mycelium texture, soluble pigment, excretions, shape and size of conidiophores, vesicles, metulae, phialides, conidia, and their texture and color, following the identification system previously used for genus Aspergillus (Raper \& Fennell 1973, Christensen 1981, Klich \& Pitt 1988, Domsch et al. 1993, Klich 2002, Samson \& Varga 2007).

Culture conditions - The strains were cultivated on $150 \mathrm{~mL}$ of Czapek-Dox (CD) (Difco Laboratories, Le Point de Claix, France) in $500 \mathrm{~mL}$ Erlenmeyer flasks. Only four were used for the microbiological reference strains, employed as a pattern and incubated at $25^{\circ} \mathrm{C}$ for seven days without agitation.

Extraction of FAs - The FA extraction was done according to Stahl and Klug (1996), with some modifications. Fungal biomass was harvested through filtration by Whatman $\mathrm{N}^{\circ} 1$ filter paper (Whatman Inc., New Jersey, USA), washed three times with distilled water $(100 \mathrm{~mL}$ 
each) and dried at $60^{\circ} \mathrm{C}$ during $2 \mathrm{~h}$. Fungal biomass $(1 \mathrm{~g})$ was then placed into $4 \mathrm{~mL}$ of saponification reagent $(150 \mathrm{~g}$ of $\mathrm{NaOH}$ in $1000 \mathrm{~mL}$ methanol) and homogenized. The homogenate was saponified in $100^{\circ} \mathrm{C}$ water for $30 \mathrm{~min}$ and cooled in a RT water bath. Then, $8 \mathrm{~mL}$ of $6 \mathrm{~N} \mathrm{HCl}$ in methanol were added to promote the formation of FA methyl esters from the aqueous phase. Samples were then placed in an $80^{\circ} \mathrm{C}$ water bath for $10 \mathrm{~min}$ and then immediately cooled to RT. Next, to extract FA methyl esters from the aqueous phase, $\mathrm{NaCl}$ saturated solution $(12 \mathrm{~mL})$ and hexane $(1 \mathrm{~mL})$ was added and then vigorously shaken. Finally, the hexanic phase (top of tube) containing the FA esters was removed and placed in a gas chromatography vial. When necessary, the samples were stored over two weeks at $-20^{\circ} \mathrm{C}$.

Analysis of FAs - FA extracts were analyzed by gasliquid chromatography (Chrompack CP 9002) equipped with a non polar fused silica capillary column $(100 \mathrm{~m}$ x $0.25 \mathrm{~mm}$ x $0.25 \mathrm{~mm}$ inside diameter; Chrompack CP-SIL 88) and flame ionization detector (FID). Data from the chromatographic analysis was sent to the computer system where FAs were identified on the basis of their retention times compared to 37 FA known standards (Sigma, Lipid Standard, Fatty Acid Methyl Ester Mixture $\# 189-19)$. They were quantified in relation to other FAs in the sample on the basis of width peak and area data.

Samples were run through the gas chromatography column for $45 \mathrm{~min}$, time enough for long-chain FAs (up to 28 carbons) to pass through. The initial oven temperature was $170^{\circ} \mathrm{C}$ and the final oven temperature of $210^{\circ} \mathrm{C}$ was reached 26 min after injection. The injector (split $1: 100$ ) temperature was $250^{\circ} \mathrm{C}$ and the detector FID temperature was $280^{\circ} \mathrm{C}$. The flow rates of the carrier gases nitrogen, hydrogen and air were 30,30 and $300 \mathrm{ml} \mathrm{min}^{-1}$, respectively.

Composition of fungal FA - Analysis principal component (APC) were used to examine relationships among all the analyzed Aspergillus spp. to indicate the most-similar FA compositions. New variables have been raised as linear combinations of the initial variables and the results were graphically plotted on two dimensions, i.e. a PC1-PC2 plot. The FA of the most similar species was then compared by an index of similarity (IS) and a multivariate statistical test (Stahl \& Klug 1996). The similarity was confirmed when the index varied be- tween $0.90-1$. To quantify the similarity of FA composition of Aspergillus spp., an index-of-relationship value was calculated using the following formula:

$$
\mathrm{IS}_{\mathrm{xy}}=\left(\mathrm{C}_{\mathrm{x}} / \mathrm{C}_{\mathrm{y}}\right)_{1}\left(\mathrm{C}_{\mathrm{x}}+\mathrm{C}_{\mathrm{y}} / 200\right)_{1}+\ldots+\left(\mathrm{C}_{\mathrm{x}} / \mathrm{C}_{\mathrm{y}}\right)_{\mathrm{n}}\left(\mathrm{C}_{\mathrm{x}}+\mathrm{C}_{\mathrm{y}} / 200\right)_{\mathrm{n}}
$$

where $\mathrm{x}$ and $\mathrm{y}$ represent the fungi being compared, $\mathrm{C}$ is the mean FA concentration expressed as a percentage of the total content for 1 through $n$ FA and $\left(\mathrm{C}_{\mathrm{x}} / \mathrm{C}_{\mathrm{y}}\right)$ represents the minor ratio of the FA used on this calculation (Stahl \& Klug 1996). To determine if Aspergillus spp. FA composition were statistically different, the Hotelling-Lawley test was used, with the species $\mathrm{x}\left(\mathrm{fa}_{1}\right.$, $\left.\mathrm{fa}_{2} \ldots \mathrm{fa}_{\mathrm{n}}\right)=\mathrm{y}\left(\mathrm{fa}_{1}, \mathrm{fa}_{2} \ldots \mathrm{fa}_{\mathrm{n}}\right)$, where fa is FA.

\section{RESULTS}

The number of FAs profiles obtained by the extraction process was higher than the ones found for Aspergillus species by Blomquist et al. (1992), Stahl and Klug (1996) and Nemec et al. (1997). The analyzed fungi contained FA of carbon chain lengths ranging from 12-20. The most common and abundant FA extracted were palmitic acid (C16:0), estearic acid (C18:0), oleic acid (C18:1) and linoleic acid (C18:2), which often corresponded to $95 \%$ of the Aspergillus spp. total FA content (Tables I, II). These four FA were present in all of the fungi examined. Others FAs: palmitoleic acid (C16:0), margaric acid (C17:0), linoleic acid (C18:3) and araquidic acid (C20:0) were commonly detected, but usually represented less than $5 \%$ of the total FA content.

Some species of Aspergillus presented different quantities and profiles of FA and also differed on the relative concentrations of each type (Tables I, II). The number of extracted FA ranged from 6-8. The relative concentration of an individual FA ranged from less than $1 \%$ of the total FA content to as much as $51 \%$.

IS was estimated to quantify the similarities among six Aspergillus spp. FA compositions. All the comparisons were statistically significant $(\mathrm{p}<0.001)$. A. ochraceus and $A$. nomius presented the highest similarity index (0.92), whereas $A$. ochraceus and A. flavus presented the lowest similarity index (0.63) (Table III).

APC was performed using the FA profiles from six Aspergillus spp. as variables. It allowed the first two APCs ( $75 \%$ of data's total variance) to enroll. The graphic dispersion, based on relative scores of the first two APCs, shows the differences between the most similar

TABLE I

Fatty acid composition of reference Aspergillus species obtained from a culture collection

\begin{tabular}{lcccccccc}
\hline & \multicolumn{7}{c}{ Percentage of total fatty acid content (\%) ${ }^{a}$} \\
\cline { 2 - 9 } Species & $\mathrm{C} 16: 0$ & $\mathrm{C} 16: 1$ & $\mathrm{C} 17: 0$ & $\mathrm{C} 18: 0$ & $\mathrm{C} 18: 1$ & $\mathrm{C} 18: 2$ & $\mathrm{C} 18: 3$ & $\mathrm{C} 20: 0$ \\
\hline A. caelatus & $15.28 \pm 0.16$ & $0.15 \pm 0.26$ & $0.19 \pm 0.32$ & $8.05 \pm 1.07$ & $20.50 \pm 4.88$ & $51.79 \pm 2.32$ & $4.04 \pm 1.25$ & $\mathrm{~T}$ \\
A. parasiticus & $16.04 \pm 0.40$ & $0.15 \pm 0.26$ & $0.18 \pm 0.31$ & $12.60 \pm 2.70$ & $21.52 \pm 6.38$ & $45.89 \pm 5.63$ & $2.52 \pm 1.81$ & $1.09 \pm 1.14$ \\
A. fumigatus & $18.48 \pm 0.70$ & $0.38 \pm 0.38$ & $0.94 \pm 0.94$ & $6.37 \pm 1.30$ & $24.14 \pm 4.21$ & $49.70 \pm 4.30$ & $\mathrm{~T}$ & $\mathrm{~T}$ \\
A. nomius & $19.05 \pm 0.82$ & $1.79 \pm 0.21$ & $\mathrm{~T}$ & $6.09 \pm 0.69$ & $31.59 \pm 3.25$ & $39.35 \pm 3.49$ & $0.33 \pm 0.57$ & $1.51 \pm 1.00$ \\
A. ochraceus & $20.20 \pm 0.92$ & $0.33 \pm 0.57$ & $\mathrm{~T}$ & $7.61 \pm 0.82$ & $33.31 \pm 1.25$ & $37.33 \pm 0.99$ & $0.62 \pm 0.63$ & $0.31 \pm 0.54$ \\
A. flavus & $14.97 \pm 1.37$ & $0.54 \pm 0.67$ & $0.25 \pm 0.33$ & $10.82 \pm 1.85$ & $35.82 \pm 4.78$ & $34.26 \pm 4.94$ & $0.84 \pm 0.71$ & $0.69 \pm 0.32$ \\
\hline
\end{tabular}

$a$ : means \pm standard deviation; $\mathrm{T}$ : trace (less than $0.15 \%$ ). 
TABLE II

Fatty acid composition of Aspergillus species obtained from maize and poultry feed

\begin{tabular}{lcccccccc}
\hline & \multicolumn{7}{c}{ Percentage of total fatty acid content (\%) } \\
\cline { 2 - 9 } Species & $\mathrm{C} 16: 0$ & $\mathrm{C} 16: 1$ & $\mathrm{C} 17: 0$ & $\mathrm{C} 18: 0$ & $\mathrm{C} 18: 1$ & $\mathrm{C} 18: 2$ & $\mathrm{C} 18: 3$ & $\mathrm{C} 20: 0$ \\
\hline A. flavus & $13.91 \pm 1.37$ & $0.73 \pm 0.47$ & $0.34 \pm 0.22$ & $11.82 \pm 1.54$ & $34.81 \pm 3.71$ & $35.32 \pm 3.95$ & $0.90 \pm 0.81$ & $0.70 \pm 0.21$ \\
A. parasiticus & $14.31 \pm 0.50$ & $0.15 \pm 0.23$ & $0.18 \pm 0.33$ & $13.80 \pm 2.60$ & $29.01 \pm 3678$ & $39.83 \pm 4.60$ & $2.22 \pm 1.11$ & $0.90 \pm 0.17$ \\
A. tamarii & $16.92 \pm 1.64$ & $0.40 \pm 0.45$ & $0.41 \pm 0.34$ & $7.94 \pm 1.23$ & $32.04 \pm 2.06$ & $42.09 \pm 2.97$ & nd & nd \\
\hline
\end{tabular}

a: means \pm standard deviation; nd: not detected.

TABLE III

Comparison of six Aspergillus species based on fatty acid profiles for multivariated test

\begin{tabular}{lccccc}
\hline & \multicolumn{5}{c}{ Relationship Index $^{a}$} \\
\cline { 2 - 6 } Species & A. caelatus & A. parasiticus & A. fumigatus & A. nomius & A. ochraceus \\
\hline A. caelatus & 1 & & & & A. flavus \\
A. parasiticus & 0.87 & 1 & & & \\
A. fumigatus & 0.88 & 0.84 & 1 & 1 & 1 \\
A. nomius & 0.71 & 0.75 & 0.81 & 0.92 & 0.63 \\
A. ochraceus & 0.70 & 0.73 & 0.77 & 0.64 & 1 \\
A. flavus & 0.84 & 0.86 & 0.79 & & \\
\hline
\end{tabular}

$a$ : significant values at the probability level of $<0.001$ by Hotelling-Lowley multivariated test.
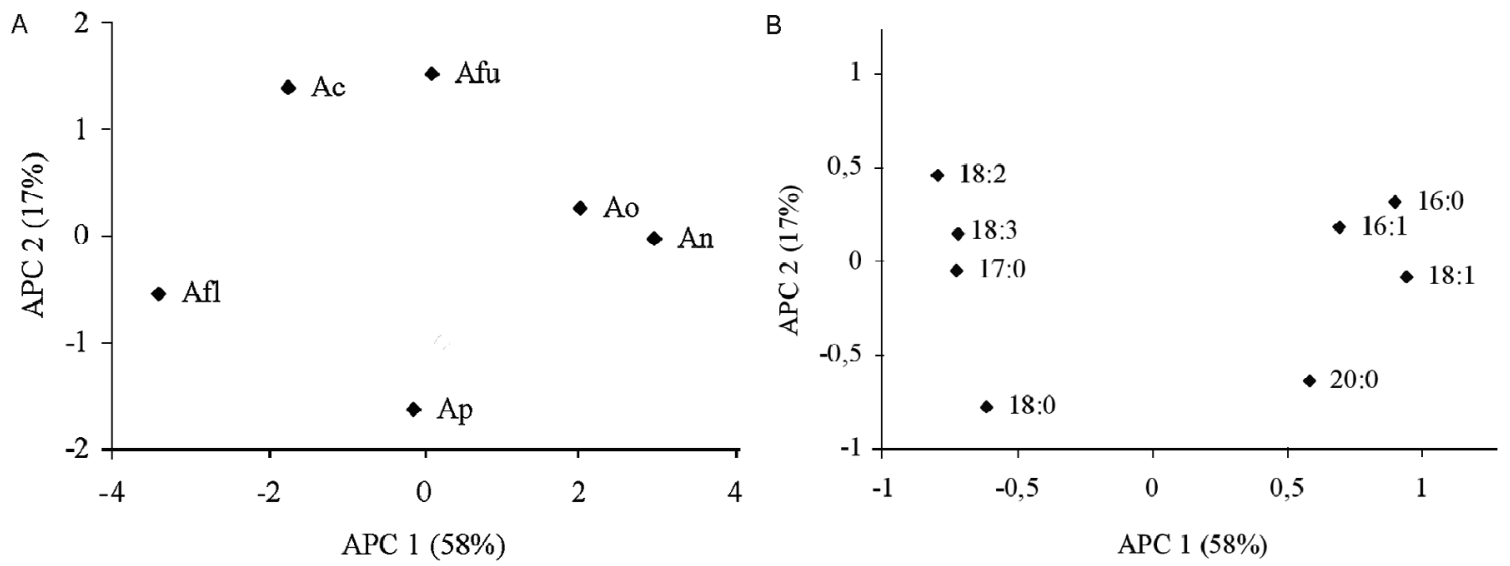

Analysis of principal components (APC) of Aspergillus species. Each species was represented by four entries in the APC, corresponding to different extractions from the same species. A: graphic representation of the fungi or a plan defined by the first two axes (APC1 and APC2) of the principal components; Ac: A. caelatus; Afl: A. flavus; Afu: A. fumigatus; An: A. nomius; Ao: A. ochraceus; Ap: A. parasiticus; B: graphic representation of the variables (fatty acid composition) or the same plan.

species (A. ochraceus and A. nomius) (Fig. 1A). Fig. 1A also presents the difference among Aspergillus spp. profiles from Section Flavi and four differentiated groups (A. caelatus and A. fumigatus), (A. flavus), (A. parasiticus) and (A. ochraceus and $A$. nomius). Fig. 1B shows the distribution of eight FA and demonstrates the presence of four distinct groups (C18:2, C18:3, C17:0), (C18:0), (C20:0) and (C16:0, C16:1, C18:1).

\section{DISCUSSION}

In this study, APC was used to discriminate fungal species belonging to Aspergillus genus, based on the similarity of FA composition. The medium used during the experiments was totally free of FA residues and allowed for an excellent biomass production in less contaminating agents (Blomquist et al. 1992).

The use of type culture as a microbiological reference strain for the morphological and FA studies employed in our work was also reported in other studies (Blomquist et al. 1992, Nemec et al. 1997, Silva et al. 1998) and was not presented by Stahl and Klug (1996) or Müller et al. (1994).

The number of FA obtained in this work was higher than the ones obtained for different fungal species reported by Blomquist et al. (1992), Stahl and Klug (1996) and Nemec et al. (1997). The capillary column length (100 m) probably favored the fungal biomass FA detection in 
comparison to the $20 \mathrm{~mL}$ capillary columns used in other studies (Blomquist et al. 1992, Müller et al. 1994, Nemec et al. 1997, Stahl \& Klug 1996, Silva et al. 1998).

In the present work, $37 \mathrm{FA}$ were analyzed. Eight types were represented the most, with palmitic acid, estearic acid, oleic acid, and linoleic acid as the most abundant, whereas palmitoleic acid margaric acid, linoleic acid and araquidic acid were detected in smaller ratios among the six evaluated species. These results were not in agreement with Nemec et al. (1997), who investigated the FA profiles of A. flavus and A. fumigatus. They reported that palmitic acid, palmitolelic acid, margaric acid, estearic acid, oleic acid, linoleic acid and linolenic acid were prevalent and araquidic acid was not found.

The APC showed similar results as those reported by other researchers (Blomquist et al. 1992, Müller et al. 1994, Stahl \& Klug 1996, Silva et al. 1998). Eight fatty acids among 37 ones found were considered to be more representative: palmitic acid (C16:0), stearic acid (C18:0), oleic acid (C18:1) and linoleic acid (C18:2). Palmitoleic acid (C16:1), margaric acid (C17:0), linolenic acid (C18:3) and araquidic acid (C20:0), which were not always detected among the six species assessed, were found at lower ratios.

The two species isolated from corn and poultry feed, A. flavus and A. parasiticus, presented FA profiles very similar to those of microbiological reference strains, which were used as a pattern for FA studies. The FA found in $A$. flavus in the present study was C16:0, C16:1, C17:0, C18:0, C18:1, C18:2, C18:3 and C20:0. These results differ from those obtained by Nemec et al. (1997), that did not observe the presence of the FA C20:0. Regarding A. tamarii, which is also isolated from corn and poultry feed, we did not find the FA C18:3 and C20:0. This consequently aided the distinction among the Aspergillus spp used as a pattern.

It was possible to verify the presence of four different groups: A. caelatus, A. fumigatus/A. flavus/A. parasiticus/A. ochraceus, A. nomius. Although there was a close relationship among species in some groups, they were distinguishable following morphological criteria such as color. These results also allowed for differentiation of species belonging to Section Flavi from other species, such as A. fumigatus and A. ochraceus.

The similarity among the FA contents could be quantified using the IS. Our results showed that, in general, all species were different. The highest similarity was among A. ochraceus and A. nomius. Stahl and Klug (1996) reported the IS of fungal strains belonging to different genera and showed some differences among them, while Blomquist et al. (1992) demonstrated no similarity between A. flavus and A. fumigatus, which is in disagreement with our results.

On the other hand, A. flavus, $A$. parasiticus and $A$. nomius (that present morphological similarity) as well as $A$. caelatus and $A$. tamarii (also morphologically similar), that belong to Section Flavi, were clearly differentiated according to FAs contents. Aspergillus spp. identification can present great morphological difficulty based on results reported by several researchers (Raper Fennell 1973, Christensen 1981, Klich \& Pitt 1988, Domsch et al. 1993, Klich 2002, Samson \& Varga 2007).
Our results demons-trated the competence of FA analysis as a tool for species identification. Other studies have used the FA analysis for solving problems when morphological and physiological variations were present (Welch 1991, Blomquist et al. 1992, Stahl \& Klug 1996, Nemec et al. 1997). Therefore, the use of both morphological methods and FA analysis increases the accuracy for fungal strains identification.

The mycelial FA composition of Aspergillus spp. can be used as a genus taxonomy tool. On the basis of these findings, it is possible to discriminate closely-related species, mainly in Section Flavi. Future approaches might consider the variation of water activity and $\mathrm{pH}$ for evaluating successful methodology employed in this work under different environmental conditions.

Due to the importance of Aspergillus variable susceptibility to multiple antifungal drugs, we believe that our work may encourage the use of FA profiles as an additional tool to facilitate the differentiation and precise identification of clinically-relevant Aspergilli; consequently, fostering improved choice in appropriate antifungal therapy.

\section{REFERENCES}

Blomquist G, Anderson B, Anderson K, Brondz I 1992. Analysis of fatty acids. A new method for characterization of moulds. $J \mathrm{Mi}$ crobiol Methods 16: 59-68.

Christensen M 1981. A synoptic key and evalution of species in the Aspergillus flavus group. Mycologia 73: 1056-1084.

Domsch KH, Gams W, Anderson T-H 1993. Compedium of Soil Fungi. Academic Press, London, 860 pp.

Guarro J, Gene J, Stchigel AM 1999. Developments in fungal taxonomy. Clin Microbiol Rev 12: 454-500.

Klich MA 2002. Identification of common Aspergillus species. CBS, Utrecht, 116 pp.

Klich MA, Pitt JI 1988. A Laboratory Guide to Common Aspergillus Species and their Teleomorphs. CSIRO Division of Food Processing, North Ryde, 116 pp.

Lechevalier H, Lechevalier MP 1988. Chemotaxonomic use of lipids an overview. In C Ratledge, SG Wilkison (ed.), Microbial Lipids, Vol. I, Academic Press, London, p. 869-902.

Menyawi IE, Wogerbauer M, Burgmann H, Graninger W 2000. Identification of yeast species by fatty acid profiling as measured by gas-liquid chromatography. J Chromatogr 742: 13-24.

Moss CW 1981. Gas-liquid chromatography as an analytical tool in microbiology. J Chromatogr 203: 337-347.

Müller MM, Kantola R, Kitunen V 1994. Combining sterol and fatty acid profiles for the characterization of fungi. Mycol Res 98 : 593-603.

Nemec T, Jernec K, Cimerman A 1997. Sterols and fatty acids of different Aspergillus species. FEMS Microbiol Lett 149: 201-205.

Raper KB, Fennell DI 1973. The Genus Aspergillus. Robert E Krieger, New York, 686 pp.

Samson RA, Pitt JI 2000. Integration of Modern Taxonomic Methods for Penicillium and Aspergillus Classification. Ed HAP, Amsterdam, $510 \mathrm{pp}$.

Samson RA, Varga J 2007. Aspergillus systematics in the genomic era. CBS Fungal Biodiversity Centre, Utrecht, 206 pp. 
Silva TL, Sousa E, Pereira PT, Ferrão AM, Roseiro C 1998. Cellular fatty acid profiles for the differentiation of Penicillium species. FEMS Microbiol Lett 164: 303-310.

Stahl PD, Klug, MJ 1996. Characterization and differentiation of filamentous fungi based on fatty acid composition. Appl Environ Microbiol 62: 4136-4146.
Veys A, Callewaert W, Waelkens E, van Den-Abeele K 1989. Aplication of gas-liquid chromatography to the routine identification of non-fermentation gram-negative bacteria in clinical specimens. $J$ Clin Microbiol 27: 1538-1542.

Welch D 1991. Applications of cellular fatty acid analysis. Clin Microbiol Rev 4: 422-438. 